\title{
Crystal structure of tetraaquamonosuberatonickel(II), $\mathrm{Ni}\left(\mathrm{H}_{2} \mathrm{O}\right)_{4}\left(\mathrm{C}_{8} \mathrm{H}_{12} \mathrm{O}_{4}\right)$
}

\author{
B.-S. Zhang and Y.-Q. Zheng*
}

Ningbo University, Institute for Solid State Chemistry, Municipal Key Laboratory of Inorganic Materials Chemistry, Ningbo, Zhejiang, 315211 P. R. China

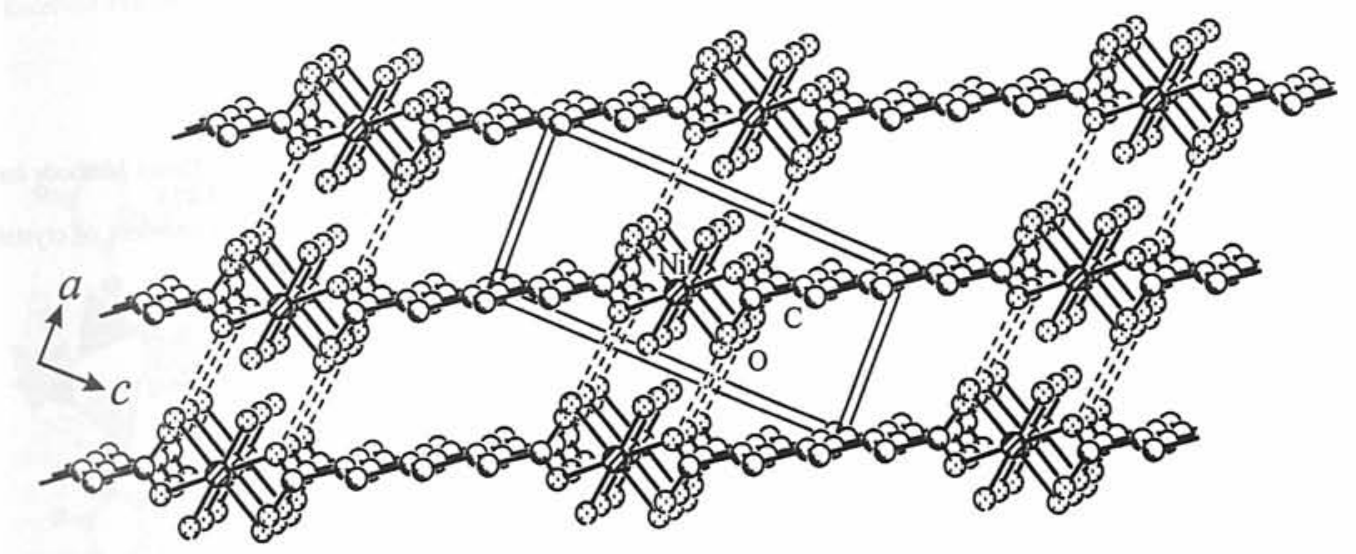

Abstract

$\mathrm{C}_{8} \mathrm{H}_{20} \mathrm{NiO}_{8}$, triclinic, $P \overline{1}$ (No. 2), $a=4.874(1) \AA, b=6.329(1) \AA$ $c=10.560(1) \AA, \alpha=76.78(1)^{\circ}, \beta=87.79(1)^{\circ}, \gamma=76.97(1)^{\circ}$, $V=308.9 \AA^{3}, Z=2, R_{\mathrm{gt}}(F)=0.024, w R_{\mathrm{ref}}\left(F^{2}\right)=0.065, T=293 \mathrm{~K}$.

Source of material

A methanolic solution of $0.44 \mathrm{~g}(2.50 \mathrm{mmol})$ suberic acid in $10 \mathrm{ml}$ $\mathrm{CH}_{3} \mathrm{OH}$ was added to a suspension of $0.30 \mathrm{~g}(2.50 \mathrm{mmol}) \mathrm{NiCO}_{3}$ in $10 \mathrm{mml} \mathrm{H}_{2} \mathrm{O}$. The resulting mixture was then stirred for one hour. After filtration, the filtrate was allowed to stand at room temperature. Green well-shaped crystals were grown by slow evaporation for about 20 days.

\section{Discussion}

The $\mathrm{Ni}$ atoms in the title compound are each coordinated by four $\mathrm{H}_{2} \mathrm{O}$ molecules and two suberato groups to complete slightly trans $\mathrm{NiO}_{6}$ octahedra with $d(\mathrm{Ni}-\mathrm{O})=2.044 \AA-2.080 \AA$. The acute cisoid $\mathrm{O}-\mathrm{Ni}-\mathrm{O}$ angles fall in the region $87.62(4)^{\circ}-$ $89.72(5)^{\circ}$ trivially less than $90^{\circ}$ while the transoid $\mathrm{O}-\mathrm{Ni}-\mathrm{O}$ angles are equal to $180^{\circ}$ due to imposition of the local 1 symmetry. The suberate anions function as bis-monodenate ligands with the middle $\mathrm{C}-\mathrm{C}$ bond centered at the crystllographic $1 c$ position. The $\mathrm{C}-\mathrm{O}$ bond to the coordinating $\mathrm{O}(1)$ atom is $1.280(2) \AA$ significantly longer than that of $1.245(2) \AA$ to the uncoordinating $\mathrm{O}(2)$ atom and the terminal $\mathrm{C}-\mathrm{C}$ bond is $1.512(2) \AA$ considerably shorter than the remaining ones averaged to $1.522 \AA$. The bis-monodenate suberato groups bridge $\mathrm{Ni}$ atoms to generate ${ }_{\infty}^{1}\left[\mathrm{Ni}\left(\mathrm{H}_{2} \mathrm{O}\right)_{4}\left(\mathrm{C}_{8} \mathrm{H}_{12} \mathrm{O}_{4}\right)_{2 / 2}\right]$ polymeric chains extending along [111] direction. The formed chain molecules display strong intramolecular hydrogen bonds between aqua $\mathrm{O}(3)$ atom and uncoordinating carboxylate $\mathrm{O}(2)^{\# 1}$ atom with $d(\mathrm{O} \cdots \mathrm{O})=2.623 \AA$ and $\angle \mathrm{O}-\mathrm{H} \cdots \mathrm{O}=157^{\circ}(\# 1=-x+1,-y,-z+1)$. The interchain hydrogen bonds with $d(\mathrm{O} \cdots \mathrm{O})=2.763 \AA-2.942 \AA$ and $\angle \mathrm{O}-\mathrm{H} \cdots \mathrm{O}=$ $157^{\circ}-170^{\circ}$ are responsible for the supramolecular assembly of the polymeric chains.

\footnotetext{
* Correspondence author (e-mail: zhengcm@nbu.edu.cn)
}

Table 1. Data collection and handling.

Crystal:

Wavelength:

$\mu$ :

Diffractometer, scan mode:

$2 \theta_{\max }$ :

$N(h k l)_{\text {measured, }} N(h k l)$ unique: Criterion for $I_{\mathrm{obs}}, N(h k l)_{\mathrm{gt}}$ :

$N(\text { param })_{\text {refined: }}$

Programs:

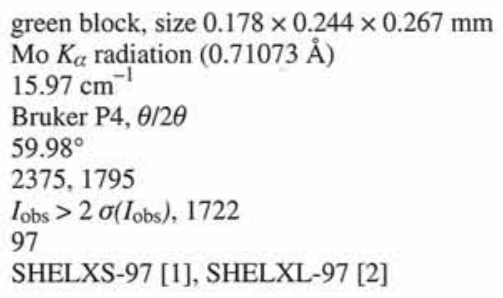

Table 2. Atomic coordinates and displacement parameters (in $\AA^{2}$ ).

\begin{tabular}{llllll}
\hline Atom & Site & $x$ & $y$ & $z$ & $U_{\text {iso }}$ \\
\hline $\mathrm{H}(2 \mathrm{~A})$ & $2 i$ & 0.2422 & -0.1148 & 0.1120 & 0.043 \\
$\mathrm{H}(2 \mathrm{~B})$ & $2 i$ & -0.0130 & -0.1572 & 0.2008 & 0.043 \\
$\mathrm{H}(3 \mathrm{~A})$ & $2 i$ & 0.1328 & -0.5414 & 0.2105 & 0.043 \\
$\mathrm{H}(3 \mathrm{~B})$ & $2 i$ & 0.3866 & -0.4972 & 0.1200 & 0.043 \\
$\mathrm{H}(4 \mathrm{~A})$ & $2 i$ & -0.1824 & -0.3195 & 0.0393 & 0.043 \\
$\mathrm{H}(4 \mathrm{~B})$ & $2 i$ & 0.0768 & -0.2949 & -0.0508 & 0.043 \\
$\mathrm{HO}(4 \mathrm{~B})$ & $2 i$ & $0.331(5)$ & $-0.293(4)$ & $0.671(2)$ & $0.042(6)$ \\
$\mathrm{HO}(4 \mathrm{~A})$ & $2 i$ & $0.143(5)$ & $-0.113(4)$ & $0.664(2)$ & $0.044(7)$ \\
$\mathrm{HO}(3 \mathrm{~B})$ & $2 i$ & $0.228(6)$ & $0.348(5)$ & $0.545(3)$ & $0.064(8)$ \\
$\mathrm{HO}(3 \mathrm{~A})$ & $2 i$ & $0.053(7)$ & $0.255(6)$ & $0.501(3)$ & $0.08(1)$ \\
& & & & &
\end{tabular}


Table 3. Atomic coordinates and displacement parameters (in $\AA^{2}$ ).

\begin{tabular}{|c|c|c|c|c|c|c|c|c|c|c|}
\hline Atom & Site & $x$ & $y$ & $z$ & $U_{11}$ & $U_{22}$ & $U_{33}$ & $U_{12}$ & $U_{13}$ & $U_{23}$ \\
\hline $\mathrm{Ni}$ & $1 f$ & $1 / 2$ & 0 & $1 / 2$ & $0.0139(1)$ & $0.0184(1)$ & $0.0230(1)$ & $-0.00080(8)$ & $-0.00415(7)$ & $-0.01152(8)$ \\
\hline $\mathrm{O}(1)$ & $2 i$ & $0.2680(2)$ & $-0.0976(2)$ & $0.3717(1)$ & $0.0190(4)$ & $0.0304(5)$ & $0.0321(5)$ & $-0.0019(4)$ & $-0.0051(4)$ & $-0.0205(4)$ \\
\hline $\mathrm{O}(2)$ & $2 i$ & $0.5786(2)$ & $-0.3867(2)$ & $0.3267(1)$ & $0.0264(5)$ & $0.0278(5)$ & $0.0369(6)$ & $0.0014(4)$ & $-0.0099(4)$ & $-0.0194(4)$ \\
\hline $\mathrm{O}(3)$ & $2 i$ & $0.1865(2)$ & $0.2807(2)$ & $0.4842(1)$ & $0.0192(4)$ & $0.0236(4)$ & $0.0306(5)$ & $0.0011(4)$ & $-0.0048(4)$ & $-0.0135(4)$ \\
\hline $\mathrm{O}(4)$ & $2 i$ & $0.2953(2)$ & $-0.1598(2)$ & $0.6569(1)$ & $0.0231(5)$ & $0.0244(5)$ & $0.0338(6)$ & $-0.0019(4)$ & $0.0005(4)$ & $-0.0081(4)$ \\
\hline$C(1)$ & $2 i$ & $0.3571(3)$ & $-0.2403(2)$ & $0.3026(1)$ & $0.0200(5)$ & $0.0224(5)$ & $0.0243(6)$ & $-0.0068(4)$ & $-0.0015(4)$ & $-0.0119(5)$ \\
\hline $\mathrm{C}(2)$ & $2 i$ & $0.1817(3)$ & $-0.2198(2)$ & $0.1836(2)$ & $0.0320(7)$ & $0.0276(6)$ & $0.0311(7)$ & $0.0000(5)$ & $-0.0115(5)$ & $-0.0167(5)$ \\
\hline $\mathrm{C}(3)$ & $2 i$ & $0.1938(3)$ & $-0.4346(2)$ & $0.1402(1)$ & $0.0324(7)$ & $0.0269(6)$ & $0.0249(6)$ & $-0.0052(5)$ & $-0.0086(5)$ & $-0.0126(5)$ \\
\hline $\mathrm{C}(4)$ & $2 i$ & $0.0066(3)$ & $-0.3938(2)$ & $0.0208(1)$ & $0.0351(7)$ & $0.0267(6)$ & $0.0279(7)$ & $-0.0039(5)$ & $-0.0108(5)$ & $-0.0132(5)$ \\
\hline
\end{tabular}

Acknowledgments. The project was supported by the National Natural Science Foundation of China (20072022), the Excellent young Teachers Program of Moe, P. R. China (C982302), the Zhejiang Provincial Natural Science Foudation (C99034), the Ningbo Municipal Key Doctor's Funds (2003A61014), and the Ningbo Municipal Natural Science Foundation (01J201301-1). The authors also thank Mr. Jian-Li Lin for X-ray data collection.

\section{References}

1. Sheldrick, G. M.: Phase Annealing in SHELXL-90: Direct Methods for Larger Structures. Acta Crystallogr. A46 (1990) 467-473.

2. Sheldrick, G. M.: SHELXL-97. Program for the refinement of crystal structures. University of Göttingen, Germany 1997. 